\title{
POROUS SQUEEZE FILM BEARING WITH ROUGH SURFACES LUBRICATED BY A BINGHAM FLUID
}

\author{
A. WALICKA*, E. WALICKI, P. JURCZAK and J. FALICKI \\ University of Zielona Góra, Faculty of Mechanical Engineering \\ ul. Szafrana 2, 65-516 Zielona Góra, POLAND \\ E-mails: A.Walicka@ijame.uz.zgora.pl \\ E.Walicki@ijame.uz.zgora.pl \\ P.Jurczak@ibem.uz.zgora.pl \\ J.Falicki@ibem.uz.zgora.pl
}

\begin{abstract}
In the paper the effect of both bearing surfaces and the porosity of one bearing surface on the pressure distribution and load-carrying capacity of a squeeze film bearing is discussed. The equations of motion of a Bingham fluid in a bearing clearance and in a porous layer are presented. Using the Morgan-Cameron approximation and Christensen theory of rough lubrication the modified Reynolds equation is obtained. The analytical solutions of this equation for a squeeze film bearing are presented. As a result one obtains the formulae expressing pressure distribution and load-carrying capacity. A thrust radial bearing is considered as a numerical example.
\end{abstract}

Key words: viscoplastic fluid, Bingham model, thrust bearing, porous layer, Christensen roughness.

\section{Introduction}

Steady state radial flows and time-dependent squeezing flows of viscoplastic fluids are encountered in a variety of fields (Covey and Stanmore, 1981; Dai and Bird, 1981; Lipscomb and Denn, 1984). These flows are found in fabrication operations such as stamping, injection molding, and sheet forming. Also, material properties of highly viscous fluids are measured with a device called the "plastometer" which incorporates a parallel-disk squeeze flow geometry (Covey and Stanmore, 1981). In addition, such flows are encountered in lubrication systems, and there is a considerable interest as to the degree in which viscoplastic additives enhance the load-bearing capacity of a lubricant.

The flows of Newtonian fluids in the clearance of a thrust bearing with impermeable surfaces have been examined theoretically. The bearing walls have been modelled as two disks, two conical or spherical surfaces. The more general case is established by the bearing formed by two surfaces of revolution (Walicka, 1994).

Porous bearings have been widely used in industry for a long time (Bujurke et al., 1987; Etsion, 1994; Morgan and Cameron, 1957; Prakash and Vij, 1973; Shukla and Isa, 1978). Basing on the Darcy model Morgan and Cameron (1957) first presented theoretical research on these bearings.

Lately the problem of curvilinear bearings with porous walls lubricated by a Bingham fluid was taken up by Walicka (2011).

In recent years, a considerable amount of tribology research has been devoted to the study of the effect of surface roughness or geometric imperfections on hydrodynamic lubrication because the bearings surfaces, in practice, are all rough and the height of the roughness asperities may have the same order as the mean bearing clearance. Under these conditions, the surface roughness affects the bearing performance considerably.

\footnotetext{
* To whom correspondence should be addressed
} 
The work in this area has mainly been confined to impermeable surfaces. The well-established stochastic theory of hydrodynamic lubrication of rough surfaces developed by Christensen (1970) formed the basis of this paper. In a series of works (Bujurke et al., 2007; Lin, 2000; 2001; Prakash and Tiwari, 1985; Walicka 2009; 2012; Walicka and Walicki, 2002a; 2002b) the Christensen model was applied to the study of the surface roughness of various geometrical configurations.

To get a better insight into the effect of surface roughness in porous bearings, Prakash and Tiwari (1984) developed a stochastic theory of hydrodynamic lubrication of rough surfaces proposed by Christensen (1970). The modified Reynolds equation (Gurujan and Prakash, 1999) applicable to two types of directional roughness structure were used by Walicka and Walicki (2002a; 2002b) to find bearing parameters for the squeeze film between two curvilinear surfaces.

In this paper a Bingham fluid is used to describe the flow of a lubricant. The modified Reynolds equation is derived and its solution for the curvilinear thrust bearing is presented. The analysis is based on the assumption that the porous matrix consists of a system of capillaries of very small radii which allows a generalization of the Darcy law and the use of the Morgan-Cameron approximation for the flow in a porous layer. According to the Christensen stochastic model (1970), different forms of Reynolds equations are derived to take account of various types of surface roughness. Analytical solutions for the film pressure are presented for the longitudinal and circumferential roughness patterns.

\section{Derivation of the modified Reynolds equation for a viscoplastic lubricant}
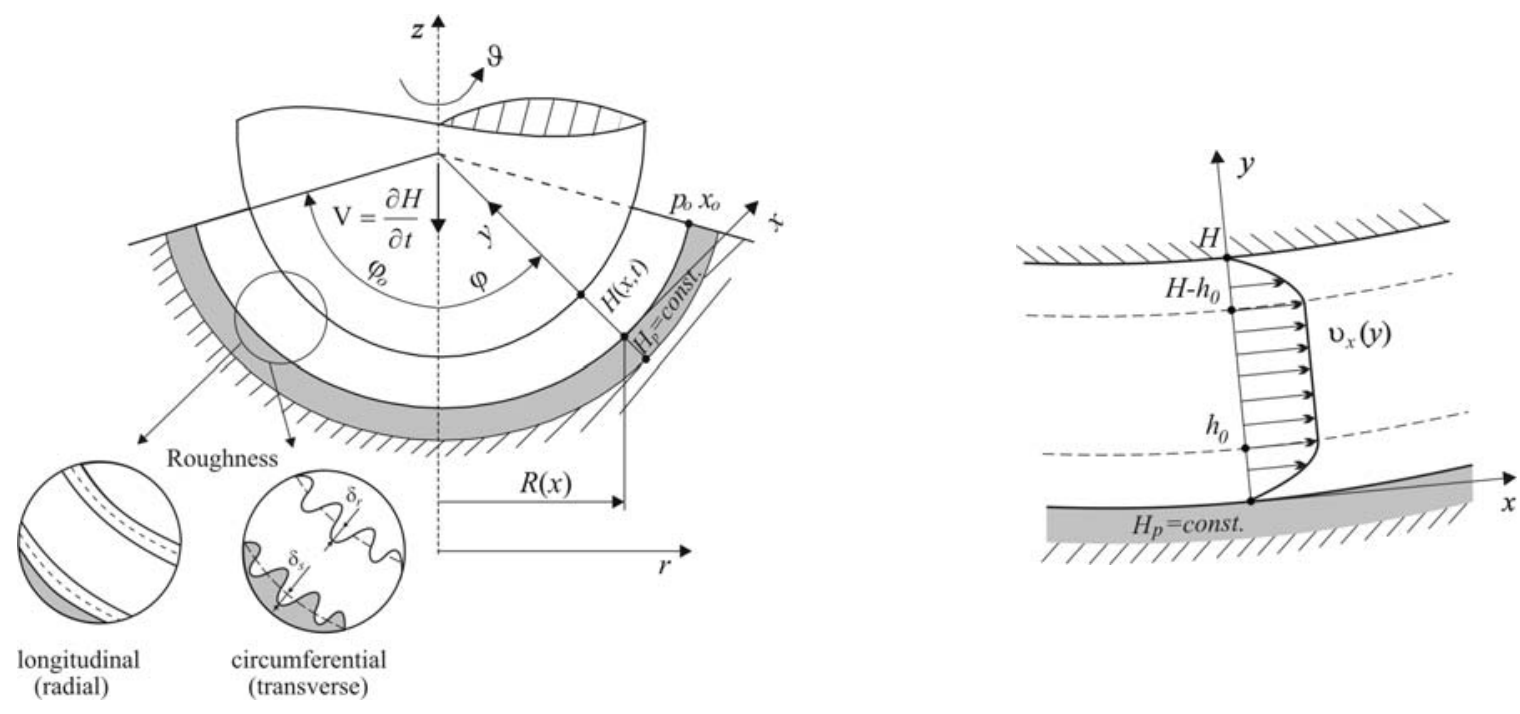

Fig.1. Configuration of a curvilinear thrust bearing with one porous wall.

Let us consider a thrust bearing with a curvilinear profile of the working surfaces shown in Fig.4. The upper bound of a porous layer is described by the function $R(x)$ which denotes the radius of this bound. The nominal bearing clearance thickness is given by the function $h(x, t)$, while the porous layer thickness is given by $H_{p}=$ const.

The expression for the film thickness is considered to be made up of two parts

$$
H=h(x, t)+h_{s}(x, \vartheta, \xi)
$$

where $h(x, t)$ represents the nominal smooth part of the film geometry, while $h_{s}=\delta_{r}+\delta_{s}$ denotes the random part resulting from the surface roughness asperities measured from the nominal level, $\xi$ describes a 
random variable which characterizes the definite roughness arrangement. An intrinsic curvilinear orthogonal coordinate system $x, \vartheta, y$ linked with the upper surface of a porous layer is also presented in Fig.1.

Taking into account the considerations of the works (Walicka, 2002; 2011; Walicki, 2005) one may present the equation of continuity and the equations of motion of a Bingham fluid for axial symmetry in the form

$$
\begin{aligned}
& \frac{1}{R} \frac{\partial\left(R v_{x}\right)}{\partial x}+\frac{\partial v_{y}}{\partial y}=0, \\
& \frac{\partial \Lambda_{x y}}{\partial y}=\frac{\partial p}{\partial x}, \quad \frac{\partial p}{\partial y}=0 .
\end{aligned}
$$

The non-zero component of the stress tensor is

$$
\Lambda_{y x}=S\left[\tau_{0}+\left|\mu \frac{\partial v_{x}}{\partial y}\right|\right]
$$

and $S=\operatorname{sgn}\left(\frac{\partial v_{x}}{\partial y}\right)$; the signum function (sgn) takes the value +1 for a positive argument $\left(\frac{\partial v_{x}}{\partial y}>0\right.$ for $\left.y \leq h_{0}\right)$ and -1 for a negative argument $\left(\frac{\partial v_{x}}{\partial y}<0\right.$ for $\left.y \leq H-h_{0}\right)$.

In the flow of fluid with yield shear stress there exists a quasi-solid core bounded by surfaces lying at

$$
y=h_{0} \quad \text { or } \quad y=H-h_{0} \quad \text { for which the shear stress is: }\left|\Lambda_{y x}\right|=\tau_{0} .
$$

The problem statement is complete after specification of boundary conditions which are

$$
\begin{array}{ll}
v_{x}(x, 0, t)=0, & v_{x}(x, H, t)=0, \\
v_{y}(x, 0, t)=V, & v_{y}(x, H, t)=\frac{\partial H}{\partial t}=\dot{H}, \\
\left.\frac{\partial p}{\partial x}\right|_{x=0}=0 & p\left(x_{o}\right)=p_{o} ;
\end{array}
$$

here $V$ is the lubricant velocity on the upper boundary of the porous matrix.

Solving Eqs (2.2)-(2.4) one obtains the following Reynolds equation [detailed solution may be found in (Walicka, 2011)]

$$
\frac{1}{R} \frac{\partial}{\partial x}\left[R h^{3}\left(-\frac{\partial p}{\partial x}\right) \Phi(\chi)\right]=-12 \mu(\dot{H}-V)
$$

where 


$$
\chi=\frac{\tau_{0}}{\tau_{w}}, \quad \tau_{w}=\frac{H}{2}\left(-\frac{\partial p}{\partial x}\right), \quad \tau_{0}=\left(\frac{H}{2}-h_{0}\right)\left(-\frac{\partial p}{\partial x}\right), \quad \chi=\frac{2 \tau_{0}}{\left(-H \frac{\partial p}{\partial x}\right)}
$$

$\tau_{w}$ - is the shear stress on the clearance wall and

$$
\Phi(\chi)=1-\frac{3}{2} \chi+\frac{1}{2} \chi^{3}
$$

Considering the porous matrix as a system of capillaries with an averaged radius $r_{c}$ and porosity $\varphi_{p}$ one may assume that the velocity components for the Bingham fluid flow in this matrix are as follows (Walicka, 2011; Walicka and Walicki, 2011)

$$
\overline{\mathrm{v}}_{x}=\Psi(\Upsilon) \Phi_{p}\left(-\frac{\partial \bar{p}}{\partial x}\right), \quad \overline{\mathrm{v}}_{y}=\Psi(\Upsilon) \Phi_{p}\left(-\frac{\partial \bar{p}}{\partial y}\right), \quad \Upsilon=\frac{2 \tau_{0}}{\left(-r_{c} \frac{\partial \bar{p}}{\partial y}\right)}
$$

where

$$
\Phi_{p}=\frac{r_{c}^{2} \varphi_{p}}{2 \mu}, \quad \Psi(\Upsilon)=\frac{1}{4}\left(1-\frac{4}{3} \Upsilon+\frac{1}{3} \Upsilon^{4}\right)
$$

$\Phi_{p}$ is the permeability of the porous matrix, $\varphi_{p}$ is the porosity.

Since the cross-velocity component $\bar{v}_{y}$ must be continuous at the porous wall-fluid interface and must be equal to $V$, we have then, by virtue of Eqs (2.6) and (2.9) $)_{2}$, the following form of the modified Reynolds equation

$$
\frac{1}{R} \frac{\partial}{\partial x}\left[R H^{3}\left(-\frac{\partial p}{\partial x}\right) \Phi(\chi)\right]=-12 \mu\left[\dot{H}-\left.\Psi(\Upsilon) \Phi_{p}\left(-\frac{\partial \bar{p}}{\partial y}\right)\right|_{y=0}\right]
$$

Using the Morgan-Cameron approximation (Morgan and Cameron, 1957) one obtains

$$
\left.\Psi(\Upsilon) \Phi_{p}\left(-\frac{\partial \bar{p}}{\partial y}\right)\right|_{y=0}=-\frac{H_{p}}{R} \frac{\partial}{\partial x}\left[R \Psi(\Upsilon) \Phi_{p}\left(-\frac{\partial \bar{p}}{\partial x}\right)\right] .
$$

When formula (2.12) is inserted into Eq.(2.11) the modified Reynolds equation takes the form

$$
\frac{1}{R} \frac{\partial}{\partial x}\left\{R\left[H^{3} \Phi(\chi)+12 \mu H_{p} \Phi_{p} \Psi(\Upsilon)\right]\left(-\frac{\partial p}{\partial x}\right)\right\}=-12 \mu \dot{H}
$$

where 


$$
\Upsilon=\chi \frac{H}{r_{c}} \quad \text { and } \quad \frac{\partial \bar{p}}{\partial x}=\frac{\partial p}{\partial x} .
$$

If the film thickness is regarded as a random quantity, a height distribution function must be associated. Many real bearing surfaces show a roughness height distribution which is closely Gaussian, at least up to three standard deviations. From a practical point of view, the Gaussian distribution is rather inconvenient and therefore a polynomial form of its approximation is chosen. Following Christensen (1970; $1971 ; 1973$ ) such a probability density function is

$$
f\left(h_{s}\right)= \begin{cases}\frac{35}{32 c^{7}}\left(c^{2}-h_{s}^{2}\right)^{3}, & -c \leq h_{s} \leq+c \\ 0, & \text { elsewhere }\end{cases}
$$

where $c$ is the half total range of the random film thickness variable. The function terminates at $c= \pm 3 \sigma$, where $\sigma$ is the standard deviation.

Inserting expected values in Eq.(2.13) we get the general form of the stochastic Reynolds equation

$$
\frac{1}{R} \frac{\partial}{\partial x}\left(E\left\{R\left[H^{3} \Phi(\chi)+12 \mu H_{p} \Phi_{p} \Psi(\Upsilon)\right]\left(-\frac{\partial p}{\partial x}\right)\right\}\right)=-12 \mu E \dot{H}
$$

where $E(\cdot)$ is the expectancy operator defined by

$$
E(\cdot)=\int_{-c}^{+c}(\cdot) f\left(h_{s}\right) d h_{s}
$$

The problem is now reduced to devising means of evaluating the left-hand side of Eq.(2.16) subject to the specific model of roughness.

The calculation of the mean film pressure distribution would require the evaluation of the expected value of various film thickness functions.

The general forms of the distribution function described by Eq.(2.17) are given in (Walicka, 2012).

\section{Solutions to the modified Reynolds equation}

Integration of Eq.(2.16) yields

$$
E\left\{R\left[H^{3} \Phi(\chi)+12 \mu H_{p} \Phi_{p} \Psi(\Upsilon)\right]\left(-\frac{\partial p}{\partial x}\right)\right\}=-12 \mu \int R E \dot{H} d x
$$

Assuming that the lubricant flow coincides with a flow with small core, then $\chi<<1, \Upsilon<<1$, and the functions $\Phi(\chi)$ and $\Psi(\Upsilon)$ become linear

$$
\Phi(\chi)=1-\frac{3}{2} \chi, \quad \Psi(\Upsilon)=\frac{1}{4}\left(1-\frac{4}{3} \Upsilon\right)
$$


Substituting Eqs (3.2) into Eq.(3.1) and taking into account relations $(2.7)_{4}$ and (2.9) 3 we reach a formula

$$
R\left[E\left(H^{3}+\frac{3}{2} H_{p} r_{c}^{2} \varphi_{p}\right)\left(-\frac{\partial E p}{\partial x}\right)-\tau_{0} E\left(3 H^{2}+4 H_{p} r_{c} \varphi_{p}\right)\right]=-12 \mu \int R E \dot{H} d x .
$$

In the present study two types of roughness structure are of interest: the longitudinal (radial) onedimensional roughness pattern, having the form of long narrow ridges and valleys running in the $x$ direction, and the circumferential (transverse) one-dimensional roughness pattern, having the form of long narrow ridges and valleys running in the $\vartheta$ direction (Walicka and Walicki, 2002a; 2002b; Walicka, 2009). For the longitudinal one-dimensional roughness

$$
H=h(x, t)+h_{s}(\vartheta, \xi)
$$

the stochastic Reynolds equation is

$$
R\left\{\left[E\left(H^{3}\right)+\frac{3}{2} H_{p} r_{c}^{2} \varphi_{p}\right]\left(-\frac{\partial E p}{\partial x}\right)-\tau_{0}\left[3 E\left(H^{2}\right)+4 H_{p} r_{c} \varphi_{p}\right]\right\}=-12 \mu \int R E \dot{H} d x
$$

but for the circumferential one-dimensional roughness

$$
H=h(x, t)+h_{s}(x, \xi)
$$

the stochastic Reynolds equation is

$$
R\left\{\left[\frac{1}{E\left(H^{-3}\right)}+\frac{3}{2} H_{p} r_{c}^{2} \varphi_{p}\right]\left(-\frac{\partial E p}{\partial x}\right)-\tau_{0}\left[\frac{3}{E\left(H^{-2}\right)}+4 H_{p} r_{c} \varphi_{p}\right]\right\}=-12 \mu \int R E \dot{H} d x
$$

Note that both Eqs (3.5) and (3.7) may be presented in one common form as follows

$$
R\left[\left(H_{j}^{(3)}+\frac{3}{2} H_{p} r_{c}^{2} \varphi_{p}\right)\left(-\frac{\partial E p}{\partial x}\right)-\tau_{0}\left(3 H_{j}^{(2)}+4 H_{p} r_{c} \varphi_{p}\right)\right]=-12 \mu \int R E \dot{H} d x
$$

where

$$
H_{j}^{(2)}=\left\{\begin{array}{lll}
E\left(H^{2}\right) & \text { for } & j=l, \\
\frac{1}{E\left(H^{-2}\right)} & \text { for } & j=c,
\end{array} \quad H_{j}^{(3)}=\left\{\begin{array}{lll}
E\left(H^{3}\right) & \text { for } & j=l, \\
\frac{1}{E\left(H^{-3}\right)} & \text { for } & j=c
\end{array}\right.\right.
$$

the case $j=l$ refers to the longitudinal one-dimensional roughness, but the case $j=c$ - to the circumferential one-dimensional roughness.

Introducing the notations

$$
M_{j}^{(2)}=3 H_{j}^{(2)}+4 H_{p} r_{c} \varphi_{p}, \quad M_{j}^{(3)}=H_{j}^{(3)}+\frac{3}{2} H_{p} r_{c}^{2} \varphi_{p},
$$


one may present the solution of Eq.(3.8) in the form

$$
E p=p_{o}+\left[F_{o}-F(x, t)\right]
$$

where

$$
\begin{aligned}
& I(x, t)=\int \frac{\int R E \dot{H} d x}{R M_{j}^{(3)}} d x, \quad J(x, t)=\int \frac{M_{j}^{(2)}}{M_{j}^{(3)}} d x, \\
& F(x, t)=12 \mu I(x, t)+\tau_{0} J(x, t), \quad F_{o}=F\left(x_{o}, t\right) ;
\end{aligned}
$$

The load-carrying capacity is defined by

$$
N=2 \pi \int_{0}^{x_{o}}\left(E p-p_{o}\right) R \cos \varphi d x
$$

the sense of angle $\varphi$ arises from Fig.1.

The calculation of the mean film pressure distribution would require the evaluation of the expected value of various film thickness functions. For the distribution function given by Eq.(2.17) we have (Walicka, 2012)

$$
\begin{aligned}
& E(H)=h, \quad E\left(H^{2}\right)=h^{2}\left(1+\frac{1}{9} Y^{2}\right), \quad E\left(H^{3}\right)=h^{3}\left(1+\frac{1}{3} Y^{2}\right), \\
& E\left(H^{-2}\right)=\frac{1}{h^{2}}\left\{1+\sum_{n=1}^{\infty} \frac{105 Y^{2 n}}{(2 n+3)(2 n+5)(2 n+7)}\right\}, \quad Y=\frac{c}{h}, \\
& E\left(H^{-3}\right)=\frac{1}{h^{3}}\left\{1+\sum_{n=1}^{\infty} \frac{105(n+1) Y^{2 n}}{(2 n+3)(2 n+5)(2 n+7)}\right\},
\end{aligned}
$$

\section{Axial squeeze film bearing} parameters

An axial squeeze film bearing is modelled by two parallel disks (Fig.2). Introducing the following

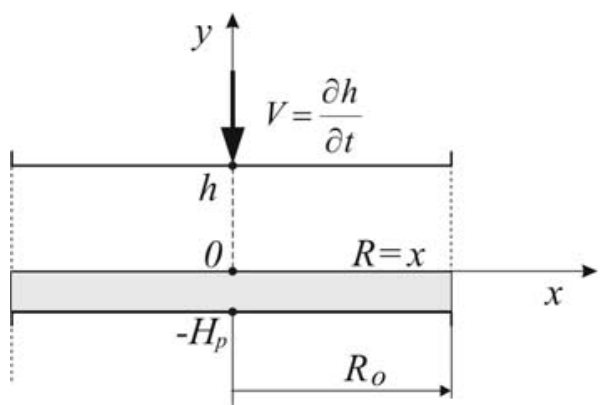

Fig.2. Axial squeeze film bearing. 


$$
\begin{aligned}
& \tilde{x}=\frac{x}{x_{o}}, \quad R=x, \quad \tilde{R}=\frac{R}{R_{o}}, \quad \tilde{h}=\frac{h}{h_{o}}=e(t), \quad e(t)=1-\varepsilon(t), \\
& K_{p}=\frac{r_{c}}{h_{o}}, \quad \tilde{H}_{p}=\frac{H_{p} \varphi_{p}}{h_{o}}, \quad \tilde{p}=\frac{\left(E p-p_{o}\right)}{\mu V_{o}} \frac{h_{o}^{3}}{x_{o}^{2}} \quad V_{o}=h_{o} \dot{\varepsilon}, \\
& \tilde{N}=\frac{N h_{o}^{3}}{\mu V_{o} x_{o}^{4}}, \quad S V=\frac{\tau_{o} h_{o}^{2}}{\mu V_{o} x_{o}},
\end{aligned}
$$

where $S V$ is the Saint-Venant plasticity number (plasticity index), one may present formulae (3.11) and (3.13) in simple non-dimensional forms

$$
\tilde{p}(\tilde{x}, t)=D\left(1-\tilde{x}^{2}\right)+E(1-\tilde{x})
$$

and

$$
\tilde{N}=\frac{\pi}{2}\left(D+\frac{2}{3} E\right)
$$

Here

$$
D=\frac{3}{\tilde{H}_{j}^{(3)}+\frac{3}{2} \tilde{H}_{p} K_{p}^{2}}, \quad E=\frac{3 \tilde{H}_{j}^{(2)}+4 \tilde{H}_{p} K_{p}}{\tilde{H}_{j}^{(3)}+\frac{3}{2} \tilde{H}_{p} K_{p}^{2}} S V
$$

and

$$
\begin{aligned}
& \tilde{H}_{j}^{(2)}= \begin{cases}e^{2}\left[1+\frac{1}{9}\left(\frac{c^{*}}{e}\right)^{2}\right] & \text { for } \quad j=l, \\
\left(\frac{1}{e^{2}}\left[1+\frac{1}{3}\left(\frac{c^{*}}{e}\right)^{2}\right]\right)^{-1} & \text { for } \quad j=c,\end{cases} \\
& \tilde{H}_{j}^{(3)}= \begin{cases}e^{3}\left[1+\frac{1}{3}\left(\frac{c^{*}}{e}\right)^{2}\right] & \text { for } \quad j=l, \\
\left(\frac{1}{e^{3}}\left[1+\frac{2}{3}\left(\frac{c^{*}}{e}\right)^{2}\right]\right)^{-1} & \text { for } \quad j=c, \quad c^{*}=\frac{c}{h_{o}} .\end{cases}
\end{aligned}
$$




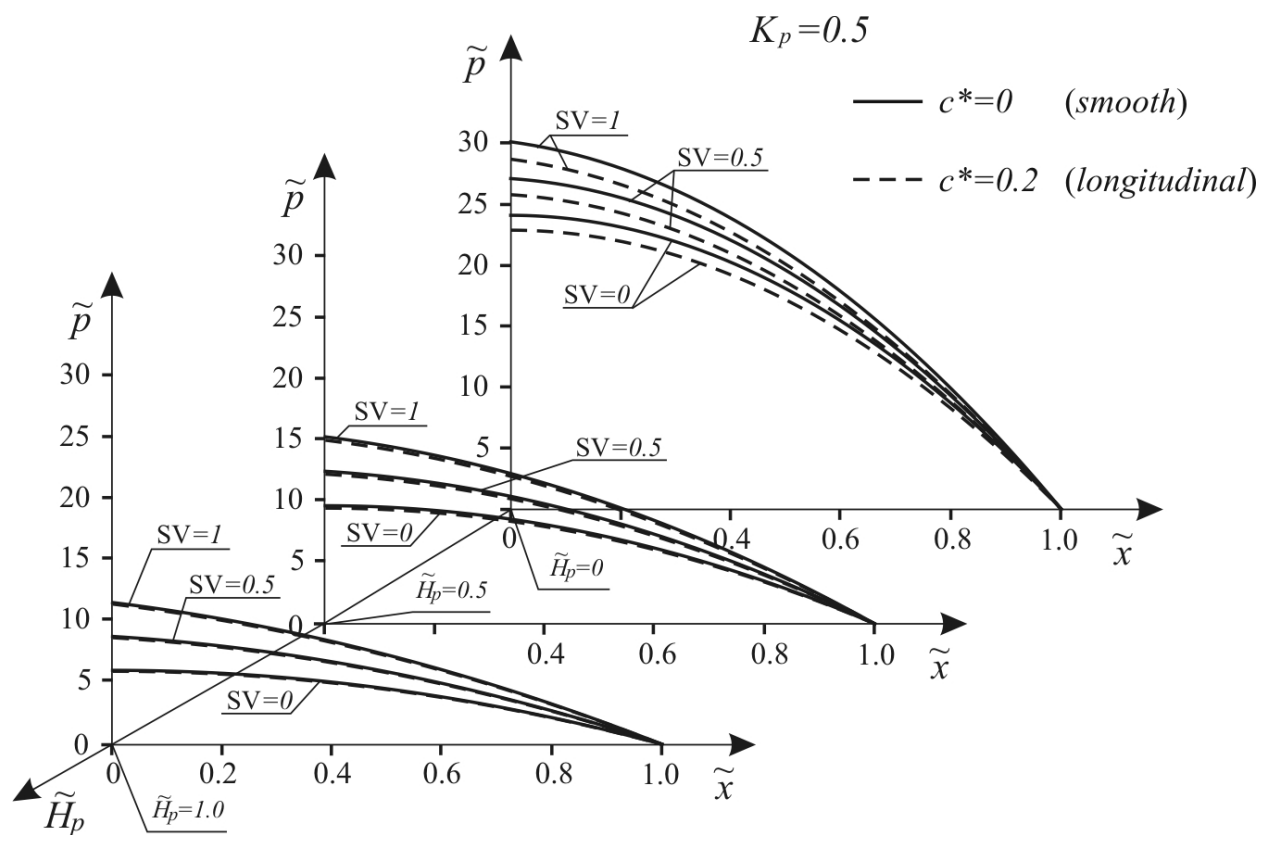

Fig.3. Dimensionless pressure distribution of the thrust bearing with rough surfaces for longitudinal roughness for $K_{p}=0.5 ; \varepsilon=0.5$ and for different values of $S V=0 ; 0.5 ; 1.0$.

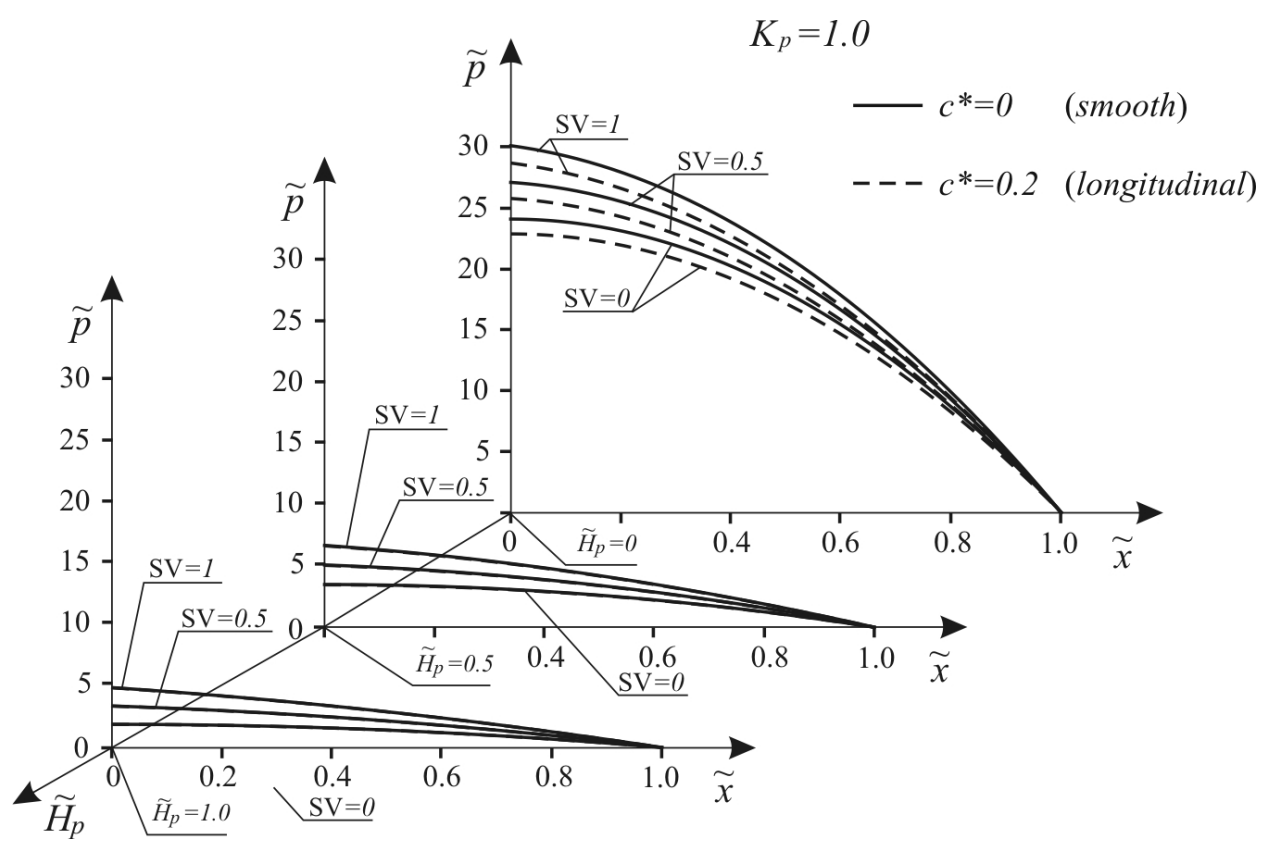

Fig.4. Dimensionless pressure distribution of the thrust bearing with rough surfaces for longitudinal roughness for $K_{p}=1.0 ; \varepsilon=0.5$ and for different values of $S V=0 ; 0.5 ; 1.0$. 


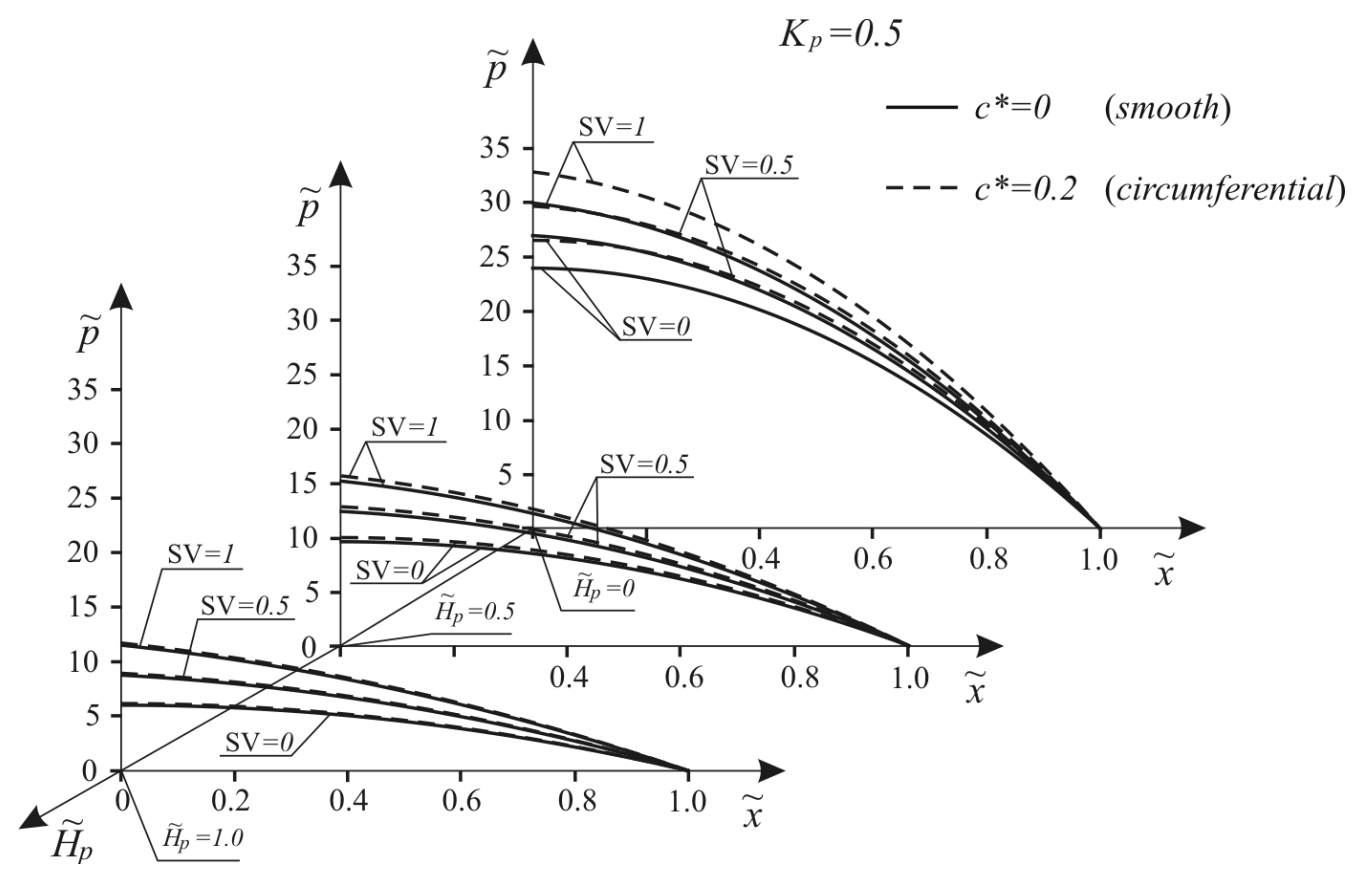

Fig.5. Dimensionless pressure distribution of the thrust bearing with rough surfaces for circumferential roughness for $K_{p}=0.5 ; \varepsilon=0.5$ and for different values of $S V=0 ; 0.5 ; 1.0$.

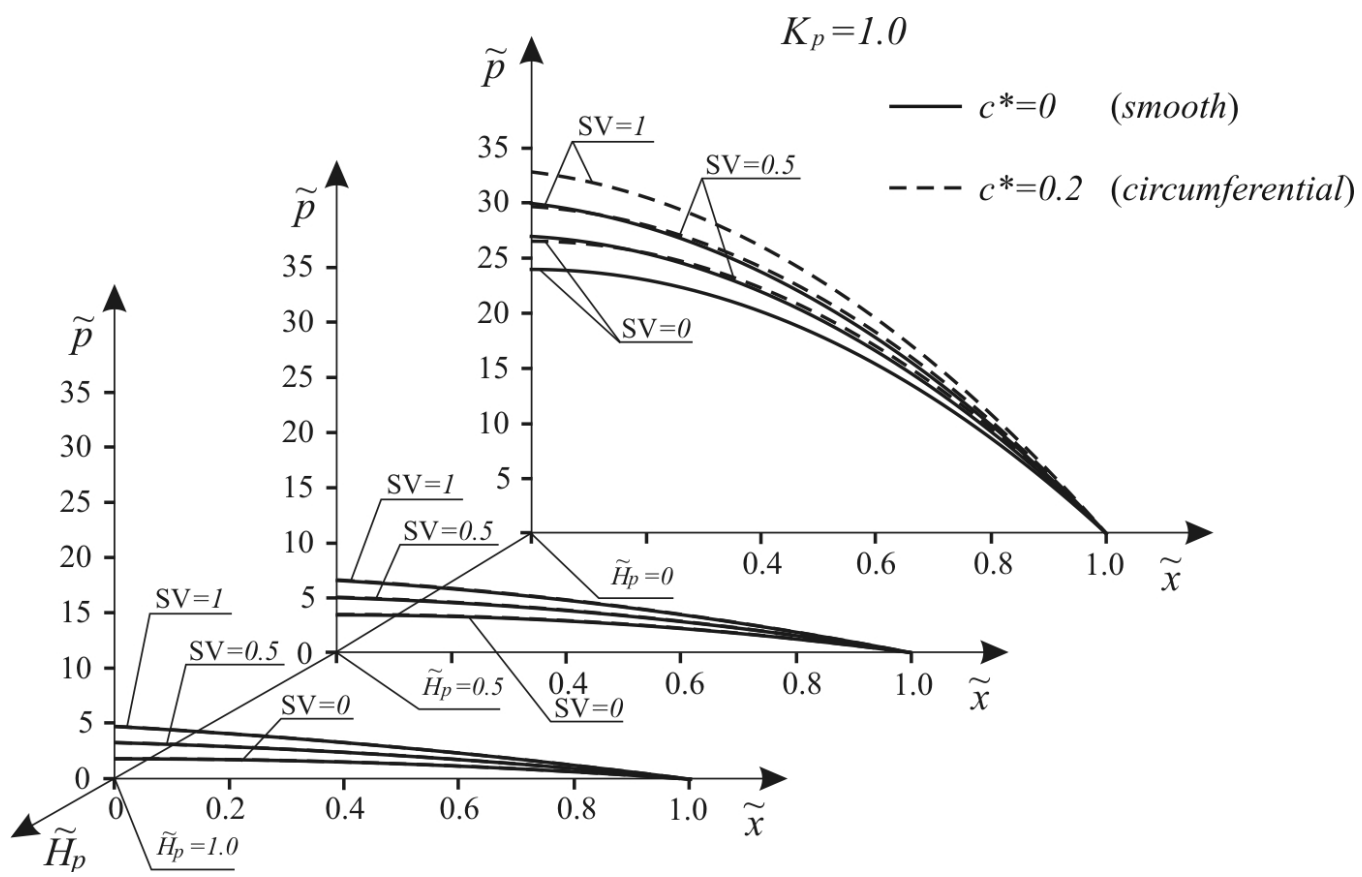

Fig.6. Dimensionless pressure distribution of the thrust bearing with rough surfaces for circumferential roughness for $K_{p}=1.0 ; \varepsilon=0.5$ and for different values of $S V=0 ; 0.5 ; 1.0$. 


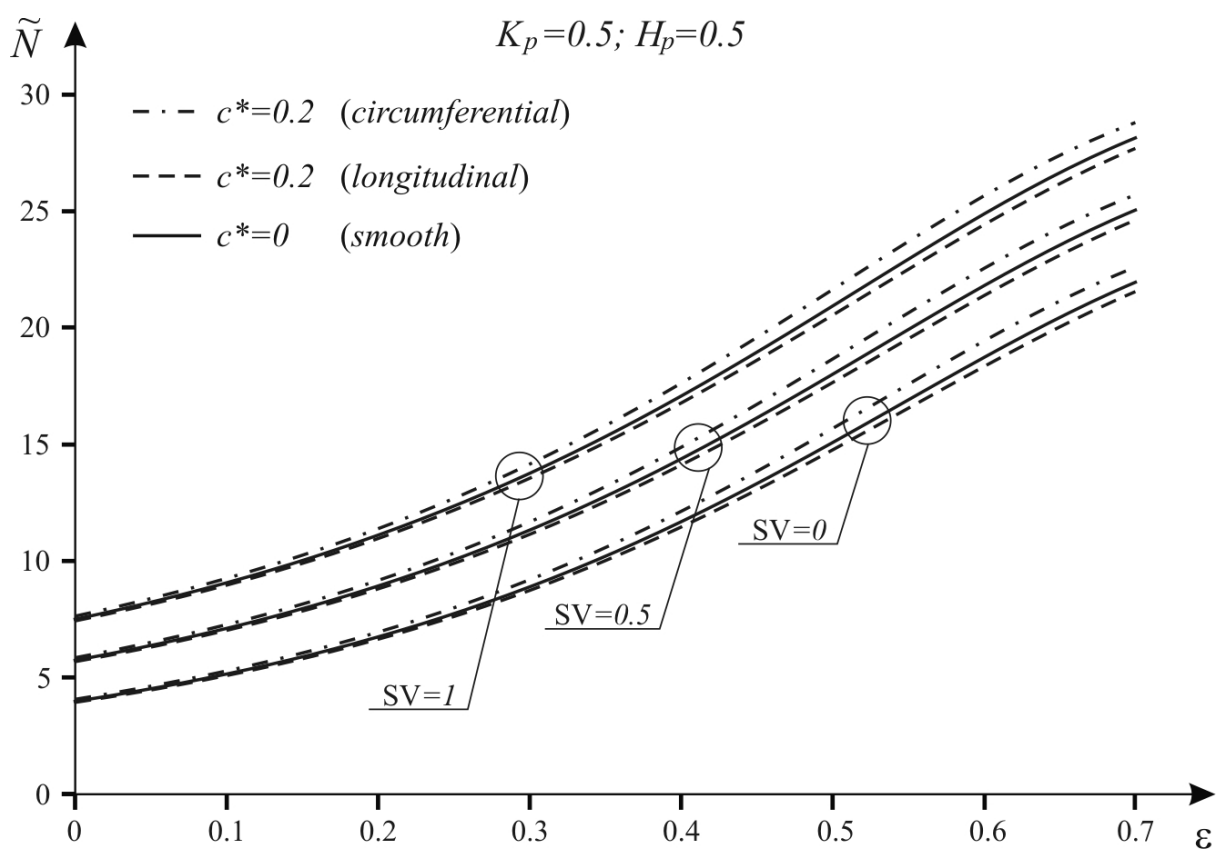

Fig.7. Load carrying capacity of the thrust bearing with rough surfaces for circumferential roughness for $K_{p}=0.5$ and for different values of $S V=0 ; 0.5 ; 1.0$.

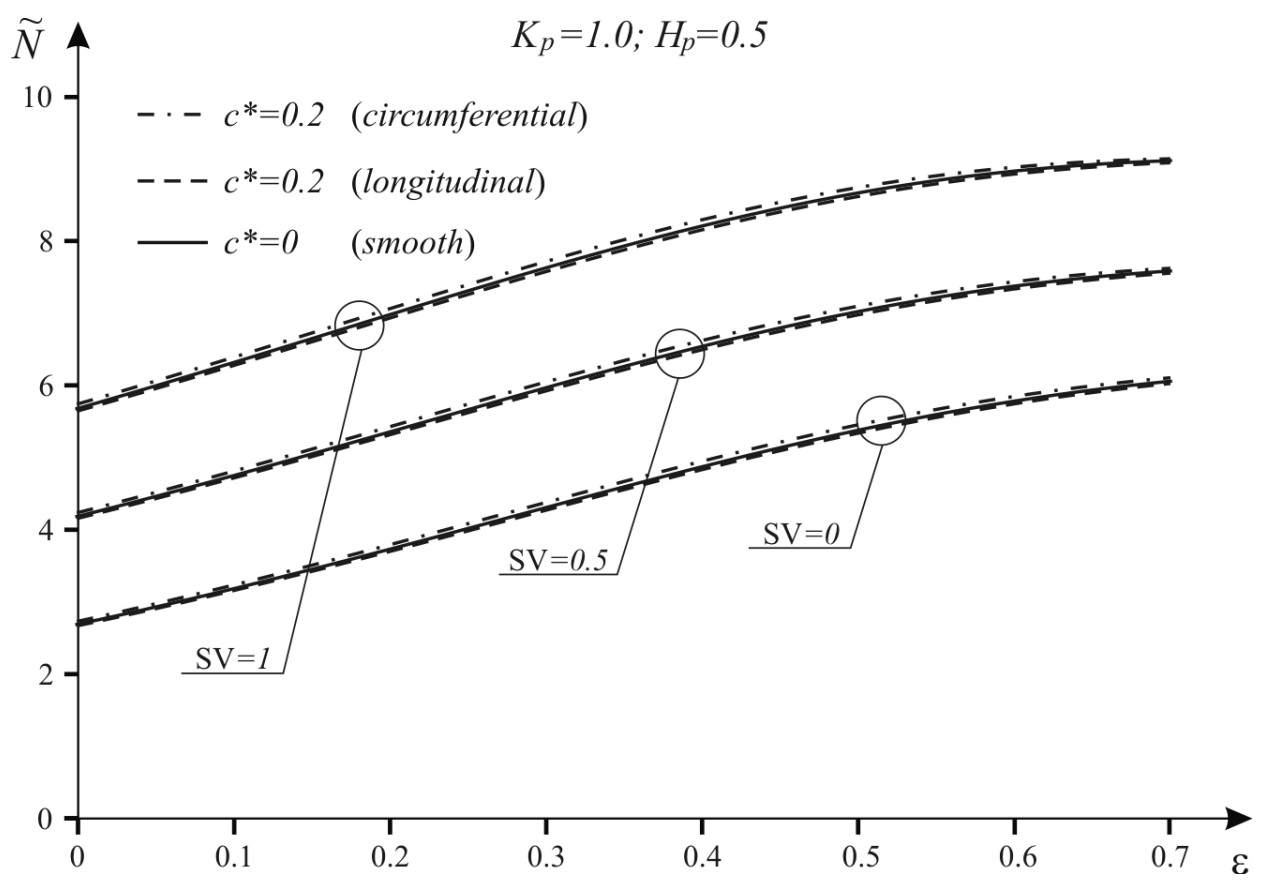

Fig.8. Load carrying capacity of the thrust bearing with rough surfaces for circumferential roughness for $K_{p}=1.0$ and for different values of $S V=0 ; 0.5 ; 1.0$. 


\section{Conclusions}

The modified Reynolds equation for a Bingham type of viscoplastic lubricants flowing in a clearance of a thrust curvilinear bearing with rough surfaces is obtained. As a result the general formulae for pressure distributions and load-carrying capacity are derived. If follows from theoretical considerations, numerical calculations and their graphic presentations that both the magnitudes are dependent on the rheological parameter $S V$ being the de Saint-Venant plasticity number and on the geometric parameters characterizing the bearing clearance and porous layer. If may be concluded that generally the pressure and load-carrying capacity increase with the increase of the values of $S V$.

Both these values decrease with the increase of porosity $K_{p}$ and thickness $\tilde{H}_{p}$ of the porous layer. The bearing surface roughness, expressed by $c^{*}$, results in some small increase of the values of bearing mechanical parameters for circumferential roughness and in a small decrease for longitudinal roughness.

\section{Nomenclature}

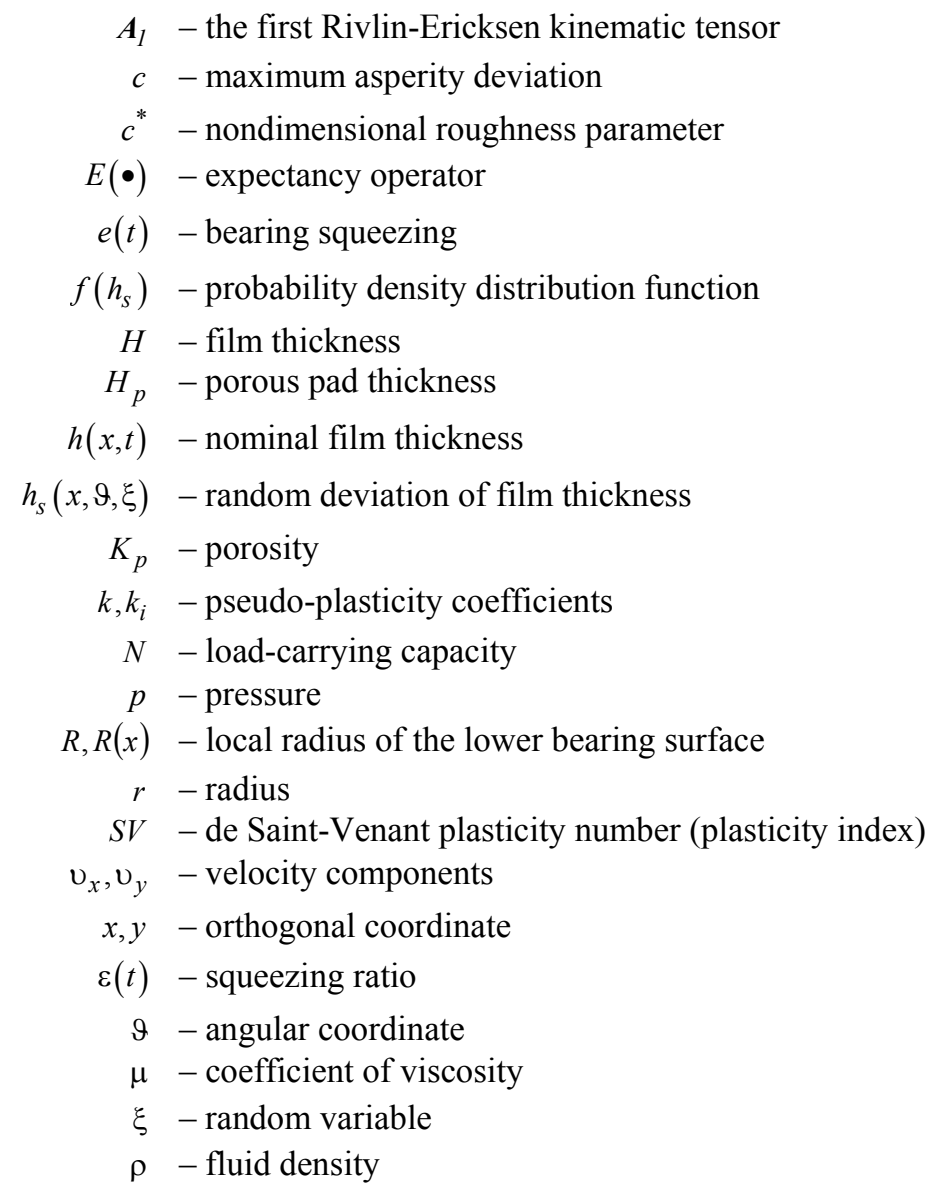

\section{References}

Bujurke N.M., Jagadee M. and Hiremath P.S. (1987): Analysis of normal stress effects in squeeze film porous bearing. Wear, vol.116, pp.237-248. 
Bujurke N.M., Kudenatti R.B. and Awati V.B. (2007): Effect of surface roughness on squeeze film poroelastic bearings with special reference to synovial joints. - Mathematical Biosciences, vol.209, pp.76-89.

Christensen H. (1970): Stochastic model for hydrodynamic lubrication of rough surfaces. - Proc. Inst. Mech. Engrs, vol.184, pt 1, pp.1013-1022.

Christensen H. and Tønder K. (1971): The hydrodynamic lubrication of rough bearing surfaces of finite width. ASME, J. Lubric. Technol., vol.93, No.2, pp.324-330.

Christensen H. and Tønder K. (1973): The hydrodynamic lubrication of rough journal bearings. - ASME, J. Lubric. Technol., vol.95, No.1, pp.166-172.

Covey G.H. and Stanmore B.R. (1981): Use of the parallel-plate plastometer for the characterisation of viscous fluids with a yield stress. - J. Non-Newtonian Fluid Mech., vol.8, pp.249-260.

Dai G. and Bird R.B. (1981): Radial flow of Bingham fluid between two fixed circular disks. - J. Non-Newtonian Fluid Mech., vol.8, pp.349-355.

Etsion I. and Michael O. (1994): Enhancing sealing and dynamic performance with partially porous mechanical face seals. - Trib. Transactions, vol.37, pp.701-710.

Gururajan K. and Prakash J. (1999): Surface roughness effects in infinitely long porous journal bearing. - Journal of Tribology, Trans. ASME, vol.121, No.1, pp.139-147.

Lin J.-R. (2000): Surfaces roughness effect on the dynamic stiffness and damping characteristics of compensated hydrostatic thrust bearings. - Int. J. Machine Tools Manufact., vol.40, pp.1671-1689.

Lin J.-R. (2001): The effect of couple stresses in the squeeze film behaviour between isotropic rough rectangular plates. - Int. J. Appl. Mech. Eng., vol.6, No.4, pp.1007-1024.

Lipscomb C.C. and Denn M.M. (1984): Flow of Bingham fluids in complex geometries. - J. Non-Newtonian Fluid Mech., vol.14, pp.337-349.

Morgan V.T. and Cameron A. (1957): Mechanism of lubrication in porous metal bearings. - Proc. Conf. on Lubrication and Wear, Inst. Mech. Eng. London, pp.151-157.

Prakash J. and Tiwari K. (1984): An analysis of the squeeze film between rough porous rectangular plates with arbitrary porous wall thickness. - Journal of Tribology, Trans. ASME, vol.106, No.2, pp.218-222.

Prakash J. and Tiwari K. (1985): Effects of surface roughness on the squeeze film between rectangular porous annular disc with arbitrary porous wall thickness. - Int. J. Mech. Sci., vol.27, No.3, pp.135-144.

Prakash J. and Vij S.K. (1973): Load capacity and time-height relations for squeeze films between porous plates. Wear, vol.24, pp.309-322.

Shukla J.B. and Isa M. (1978): Externally pressurised porous thrust bearing with power-law lubricant. - Wear, vol.33, pp.85-92.

Walicka A. (1994): Accurate and Asymptotic Solutions of Viscous Fluids in a Clearance Bounded by Two Co-axial Surfaces of Revolution (in Polish). - Warsaw: WN-T.

Walicka A. (2002): Rotational Flows of Rheologically Complex Fluids in Thin Channels (in Russian). - Zielona Gora: University Press.

Walicka A. (2009): Surface roughness effects in a curvilinear squeeze film bearing lubricated by a power-law fluid, Int. J. Appl. Mech. Engng, vol.14, No.1, pp.277-293.

Walicka A. (2011): Pressure distribution in a porous curvilinear squeeze film bearing lubricated by a Bingham fluid. Int. J. Appl. Mech. Enging, vol.16, No.4, pp.1215-1224.

Walicka A. (2012): Porous curvilinear squeeze film bearing with rough surfaces lubricated by a power-law fluid. Journal of Porous Media, vol.15, No.1, pp.29-49.

Walicka A. and Walicki E. (2002a): Surface roughness effect on the pressure distribution in curvilinear thrust bearings. - Exploitation Problems of Machines, vol. 131, No.3, pp.157-167. 
Walicka A. and Walicki E. (2002b): Couple stress and surface roughness effects in curvilinear thrust bearings, Int. J. Appl. Mech. Engng, vol.7, Spec. Issue: SITC, pp.109-117.

Walicka A. and Walicki E. (2011): Non-Newtonian fluids flows in porous media. - In: Rheology - Theory and Application, vol.2., pp.337-367, EKMA Press, Warsaw.

Walicki E. (2005): Rheodynamics of Slide Bearings Lubrication (in Polish). - Zielona Gora: University Press.

Received: July 2, 2014

Revised: August 3, 2014 